\title{
ANÁLISE DA PERIODICIDADE DOS FOLHELHOS NEGROS DO POÇO DSDP- 530 (BACIA DE ANGOLA): UM ESTUDO COMPARATIVO ENTRE A ANÁLISE ESPECTRAL UTILIZANDO HARMÔNICOS E WAVELET.
}

\author{
ARMANDO ANTONIO SCARPARO CUNHA ${ }^{1}$, NILO CHAGAS DE AZAMBUJA FILHO ${ }^{2}$
}

\begin{abstract}
A cyclostratigraphic analysis was carried out on a Cenomanian-Turonian sedimentary succession sampled in the well 530A of Deep Sea Drilling Project. This well-defined cyclic sedimentary deposit composed of marlstones, siltstones and black shalestones couplets, allowed to compare two different spectral analysis methods. The spectral analysis using Morlet Wavelet Transform performed on the colour log of the core 98 shows to be advantageous compared to the Fast Fourier Transform method. The Morlet Wavelet Transform method gives information on frequencies changes through the depth, allowing monitoring the accumulation ratio variations through time. The use of spectral analysis allowed recognizing of wavelength cycles of 19,21 e 41 $\mathrm{cm}$ in thickness, which was interpreted to be linked to variations, in the depositional conditions, induced by precession and obliquity cycles. The Wavelet method shows us that these cycles were recorded mainly at the basal part of the core.
\end{abstract}

key-word:Cyclostratigraphy, Wavelet analysis, Fast Fourier analysis.

\begin{abstract}
Resumo A análise cicloestratigráfica de depósitos sedimentares do Cenomaniano-Turoniano recuperados no testemunho 98 do poço 530A do projeto Deep Sea Drilling, formados por folhelhos, siltitos e margas que apresentam um padrão rítmico, permitiu comparar dois diferentes tipos de análise espectral. A análise espectral realizada na curva de coloração, obtida a partir das fotos do testemunho, pelo método da Transformada Wavelet de Morlet demonstrou ser vantajosa quando comparada com as análises espectrais, que utilizam a Transformada Rápida de Fourier. A principal vantagem do primeiro método é permitir verificar as variações de freqüência dos ciclos ao longo da profundidade, e desta forma monitorar mudanças na taxa de acumulação. Utilizando a análise espectral foram reconhecidos ciclos com comprimento de onda de 19,21 e $41 \mathrm{~cm}$ de espessura, interpretados como associados a variações deposicionais induzidas pelos ciclos orbitais de precessão e obliqüidade. A análise pelo método de Wavelet tornou possível concluir que esses ciclos somente foram registrados na parte basal do testemunho.
\end{abstract}

Palavras-chave:Cicloestratigrafia, Análise por Wavelet, Análise por Fourier

INTRODUÇÃo A análise espectral compreende métodos estatísticos que visam verificar a existência de periodicidade em uma série temporal. Podem ser considerados como exemplos de séries temporais em geologia os valores numéricos dos perfis geofísicos, dados geoquímicos, resultados de análises bioestratigráficas quantitativas ou valores numéricos representativos da cor de testemunhos. Enfim, qualquer propriedade física ou química que identifique o caráter oscilatório da sucessão sedimentar, medida segundo intervalos de tempo ou distâncias iguais consiste em uma série temporal. Existem vários métodos de análise espectral que se diferem pelo algoritmo utilizado e pela maneira que os resultados são apresentados. A maior parte deles utiliza a Transformada de Fourier para extrair o conteúdo de freqüência existente em uma dada série temporal (Williams, 1989; Sprenger \& Ten Kate, 1993; Sageman et al., 1997). Contudo alguns autores têm demonstrado que a utilização do algorítmico denominando de Transformada de Wavelet permite extrair o conteúdo de freqüência de uma série temporal com vantagens em relação aos métodos fundamentados na Transformada de Fourier (Prokoph \& Agterberg, 1999; Niebuhr \& Prokoph, 1997). Com intuito divulgar esse método, o presente trabalho se propõe a comparar os métodos de Transformada de Wavelet e Transformada Rápida de Fourier ou Análise de Harmônicos, demonstrando as vantagens da utilização do primeiro. Para essa finalidade foi escolhida uma série temporal que representa a sucessão rítmica de margas, siltitos e folhelhos negros do testemunho 98 do poço 530A, perfurado pelo Projeto "Deep Sea Drilling" (DSDP). Cabe destacar ainda que o presente trabalho se propõe a fazer uma abordagem mais prática da utilização do método da Transformada de Wavelet, sem entrar na conceitua- ção matemática do método, que pode ser consultada em Prokoph \& Barthelmes (1996). Na análise dos Harmônicos foi utilizado o Programa computacional Statistica ${ }^{\mathrm{TM}}$. Nas análises por Wavelet utilizou-se um programa desenvolvido pela ADDLABS da Universidade Federal Fluminense em conjunto com a PETROBRAS. A base matemática para o desenvolvimento deste programa seguiu o trabalho de Prokoph \& Barthelmes (1996).

MÉTODOS Transformada Rápida de Fourier A Transformada Rápida de Fourier (FFT), um dos dois métodos analíticos utilizados neste trabalho, parte do princípio que os padrões cíclicos de uma série temporal podem ser expressos por um somatório de funções cosenoidais-senoidais sendo cada função definida por uma amplitude, freqüência e fase. Estas funções são estatisticamente independentes, e a sua importância relativa dentro da série temporal pode ser expressa por sua variância. Os resultados das análises espectrais são expressos em um gráfico onde no eixo das abscissas encontram-se as freqüências analisadas e no eixo das ordenadas a potência espectral, que é uma função da variância da frequêencia analisada.

O método FFT calcula a potência espectral das frequiências discretas múltiplas do número de elementos da série temporal, também denominadas de harmônicos. O número máximo de frequêencias analisadas é a metade do número de elementos da série temporal. Por exemplo, em uma série temporal de 100 elementos, somente as 50 frequiências compreendidas entre 0,01 e 0,50 terão a sua variância calculada. Considerando que a freqüência é o inverso do período, os primeiros harmônicos guardam uma distância entre si maiores que os últimos. No exemplo citado acima, os três ciclos de maior periodicidade se- 
riam compostos por 100 elementos $(f=0,01), 50(f=0,02)$ e $33(f$ $=0,03)$; e os mais curtos por 2,08 elementos $(f=0,48), 2,04(f$ $=0,49)$ e $2,00(\mathrm{f}=0,50)$. Esta característica da FFT pode ser particularmente problemática em situações onde a variação na taxa de acumulação é acentuada ou em série temporais onde se deseja extrair freqüência de ordem escalar muito distinta.

A figura 1 ilustra o comportamento dos periodogramas frente às variações na taxa de acumulação. Nesta figura observam-se dois periodogramas correspondentes à análise espectral de duas sucessões hipotéticas de 81 metros que mostram um padrão de ciclicidade de estratos claros alternados com escuros (a e b). Estes estratos seguem a periodicidade de 23,41 e $100 \mathrm{ka}$. A linha senoidal (E) representa o ciclo de excentricidade $(100 \mathrm{ka})$, e a outra $(\mathrm{P}+\mathrm{O}+\mathrm{E})$ o somatório das três funções que definem os ci- clos de precessão (21 ka), obliqüidade (41 ka) e excentricidade curta (100 ka). Na primeira seção (a) a taxa de acumulação é de $1 \mathrm{~cm} / \mathrm{ka}$, constante ao longo de toda a seção, o que resulta em três ciclos bem definidos no periodograma (a). No segundo caso (b), a taxa inicial é $1 \mathrm{~cm} / \mathrm{ka}(54-81 \mathrm{~m})$, passa para $0,9(54-27$ $\mathrm{m}$ ) e atinge $0,8 \mathrm{~cm} / \mathrm{ka}(27-0 \mathrm{~m})$. No periodograma o ciclo de excentricidade encontra-se bem definido, ainda que os maiores valores da potência espectral estejam divididos entre ciclos que correspondem a 0,8 e a $1 \mathrm{~m}$. Os ciclos de obliqüidade e precessão (41 e $23 \mathrm{ka}$ ) são representados por faixas de freqüências de valores espectrais elevados. Nesta situação torna-se difícil individualizar o comprimento dos ciclos, sendo estes definidos por um valor médio.

Transformada de Wavelet de Morlet Este método foi de-

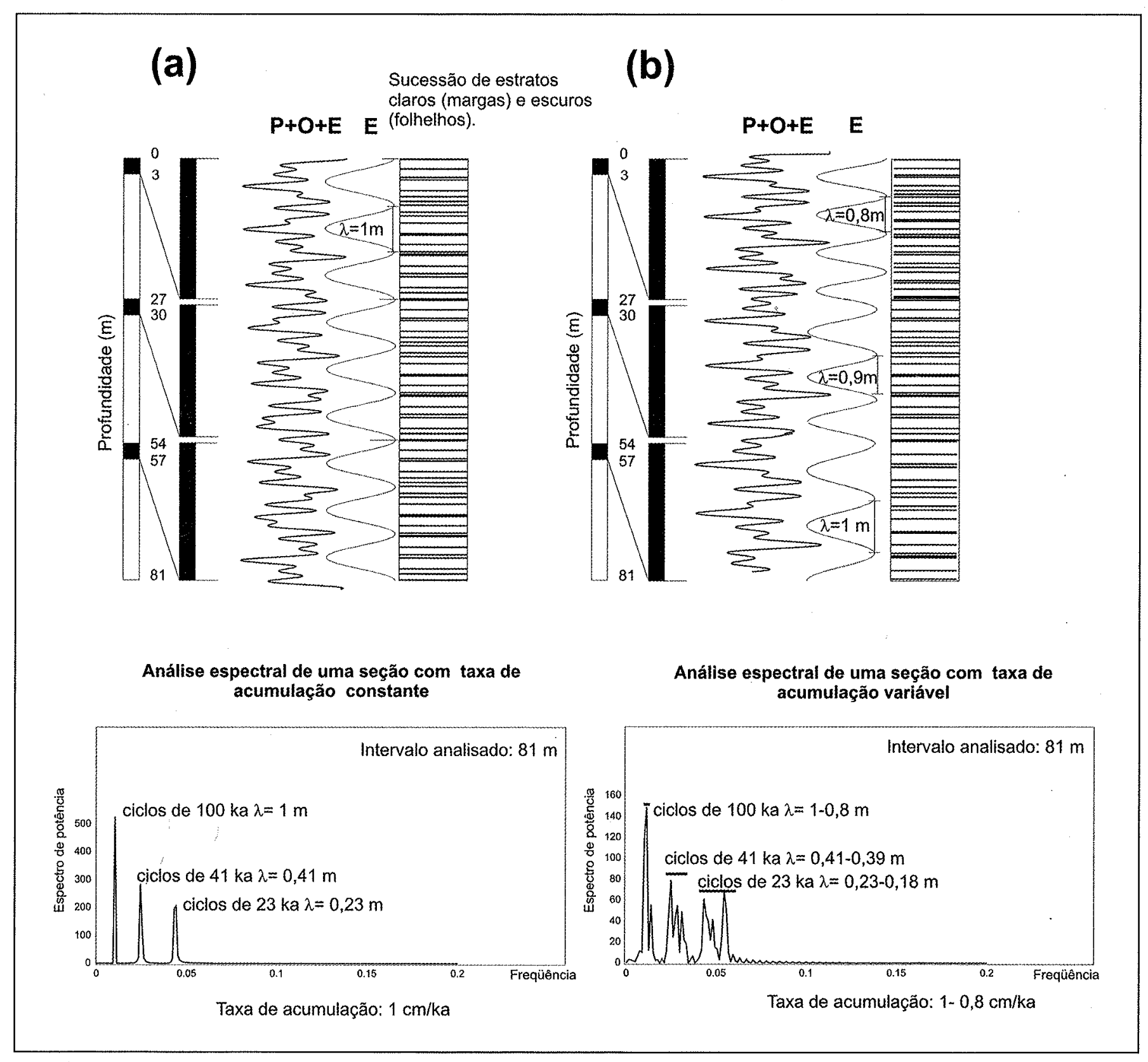

Figura 1 - Análise espectral de duas seções rítmicas hipotéticas (a e b). As linhas escuras representam camadas ricas em MO que seguem uma periodicidade de 23, 41 e $100 \mathrm{ka}$. A linha senoidal (E) representa o ciclo de excentricidade curta $(E=100 \mathrm{ka})$. A outra curva representa o somatório dos ciclos de excentricidade $(E)$, obliqüidade $(O)$ e precessão $(P)$. No primeiro caso (a), a taxa de acumulação é constante de $1 \mathrm{~cm} / \mathrm{ka}$, ao longo dos 81 metros. Os três ciclos estão perfeitamente definidos no periodograma. No segundo caso (b), a taxa de acumulação inicial é $1 \mathrm{~cm} / \mathrm{ka}(81-54 \mathrm{~m})$, passa para $0,9 \mathrm{~cm} / \mathrm{ka}(54-27 \mathrm{~m})$ e atinge 0,8 $\mathrm{cm} / \mathrm{ka}(27-0 \mathrm{~m})$. Neste caso, a periodicidade dos ciclos não pode ser estabelecida com precisão no periodograma. 
senvolvido por Prokoph e Barthelmes (1996) com o intuído monitorar as variações no conteúdo de freqüências de uma série temporal segundo a escala temporal. Ou seja, o método permite detectar quando um sinal com freqüência definida e valor espectral elevado surge e desaparece ao longo de uma série temporal. De forma similar, segundo os autores, a denominada Transformada de Fourier "janelada" também permite extrair frequiências controlando a dimensão tempo, contudo o método apresentado por eles tem a vantagem de detectar um espectro de freqüência muito mais amplo a partir do dimensionamento variável da janela de análise. $\mathrm{O}$ algorítmico desenvolvido utiliza a transformada de uma função denominada de Wavelet de Morlet, adequada para atender as especificidades das análises espectrais utilizadas em estudos cicloestratigráficos. Ao utilizar uma função básica que pode ser parametrizada durante a análise espectral, permitindo alterar o comprimento de onda, o conteúdo freqüência e amplitude, essa técnica propicia a extração de um grande espectro de freqüências. Como na prática o programa ADDSPECTRAL permite alterar somente o parâmetro (1), a seguir serão feitas algumas considerações sobre como a alteração deste parâmetro reflete na análise dos ciclos. Observe nos gráficos da figura 2,o que a alteração de (1) representa na função Wavelet de Morlet. Valores reduzidos de (1), produzem wavelets curtas, que resultam em alta resolução em tempo, mas baixa em freqüência (Fig. 2 , onde $l=5 / \pi$ ). Por outro lado, se $(l)$ tiver valores elevados (Fig. 2 , onde $1=15 / \pi)$, a resolução das freqüências será otimizada em detrimento de uma redução na resolução temporal.

Os resultados das análises espectrais utilizando wavelet são expressos por três parâmetros: periodicidade dos sinais detectados na série temporal (S); posicionamento da "onda" detectada $(\mathrm{t})$; e a intensidade do sinal detectado. Esses três parâmetros são visualizados em matrizes onde cada linha representa um valor de $(\mathrm{t})$ e cada colunas um valor de (S), como atributo de cada uma destas células tem-se um valor de $(m)$. Desta forma obtêm-se um escalograma, onde no eixo Y pode-se obter a posição média do sinal reconhecido e em X a periodicidade. Em cada célula o atributo $(\mathrm{m})$ é transformado de um valor numérico para uma cor, que representa a intensidade deste ciclo na série temporal. No programa ADDSPECTRAL essa matriz é apresentada de forma mais adequada aos estudos de séries temporais com aplicação em geologia. Na escala horizontal está a periodicidade e na vertical a profundidade. As cores expressam a intensidade dos valores espectrais, valores muito baixos ou nulos são coloridos com azul, valores moderados de verde e médios a elevados em amarelo-vermelho (Fig. 3a).

COMPARAÇÃO ENTRE OS MÉTODOS WAVELET E HARMÔNICOS A falta de controle das variações da taxa de acumulação ao longo da seção estudada é a principal desvantagem das análises espectrais que utilizam a Transformada de Fourier. Nas análises espectrais de séries temporais utilizando a Análise Rápida de Fourier parte-se do princípio de que a taxa de acumulação é constante. As variações na taxa de sedimentação tornam-se quase impossíveis de serem monitoradas devido à inexistência de métodos eficazes para reconhecer pequenos hiatos ou os efeitos da compactação. Na prática, assume-se que a taxa não varia ao longo do intervalo analisado, e utiliza-se como "taxa de sedimentação" o valor resultante da espessura da coluna sedimentar pelo tempo na qual se supõe que ela represente. Este cálculo, ao invés de expressar a taxa de sedimentação, representa na verdade a taxa mínima de acumulação ou simplesmente a taxa de acumulação de sedimentos, uma vez que o valor obtido será sempre inferior à taxa de sedimentação verdadeira.

A representação da análise espectral FFT é feita pelo periodograma, que não permite monitorar o comportamento de um determinado sinal ao longo da dimensão tempo-profundidade.

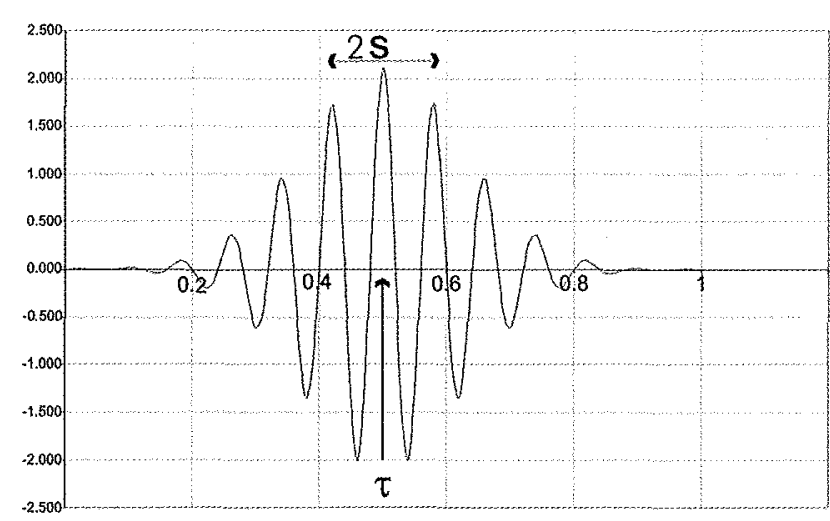

$(s)=0,08 ;(\tau)=0,5$ e $(1)=5 / \pi$

$(s)=0,08 ;(\tau)=0,5$ e $(l)=15 / \pi$

$$
\psi_{\mathrm{Re}}(s, \tau, t)=\pi^{-\frac{1}{4}}(s \cdot l)^{-\frac{1}{2}} \cos \left[2 \pi \frac{(t-\tau)}{s}\right] \exp \left[-\frac{1}{2}\left(\frac{t-\tau}{s \cdot l}\right)^{2}\right]
$$

Figura 2-Observe graficamente como a parametrização da função Wavelet de Morlet permite ora aumentar a resolução das freqüencias extraidas de uma série temporal, ora determinar com maior precisão do posicionamento do sinal na série temporal. Conforme o exemplo citado em Prokoph e Barthelmes (1996) a função Wavelet de Morlet pode ser parametrizada por (s), ( $t$ ) $e$ (l) onde (s) é o comprimento de onda, $(t)=$ posição média da onda e (l)= comprimento da wavelet. Valores mais elevados de (l) produzem "wavelets mothers" mais longas com capacidade de resolver melhor a "dimensão" freqüencia, enquanto valores mais reduzidos de (l) produzem uma "waveletes mothers" mais curta que possibilitam um melhor posicionamento dos sinais detectados na série temporal. 
Logo, os sinais periódicos com pouca expressão em termos de tempo-profundidade e sinais presentes ao longo de toda a seção não serão discriminados. As mudanças nas periodicidades dos sinais principais induzidas por alterações nas taxas de acumulação também não podem ser reconhecidas. Se o periodograma apontar para ciclos mal-definidos, a caracterização da origem destes ciclos torna-se extremamente difícil. Nesta situação, seria necessário, redirecionar subjetivamente sucessivas análises em diferentes intervalos de profundidade, até a obtenção de intervalos onde ciclos bem definidos pudessem ser encontrados.

A wavelet, ao contrário da transformada rápida de Fourier, não expressa seus resultados através de periodogramas, mas sim através de escalogramas. Utilizando o escalograma é possível monitorar o comportamento dos ciclos ao longo da profundi-

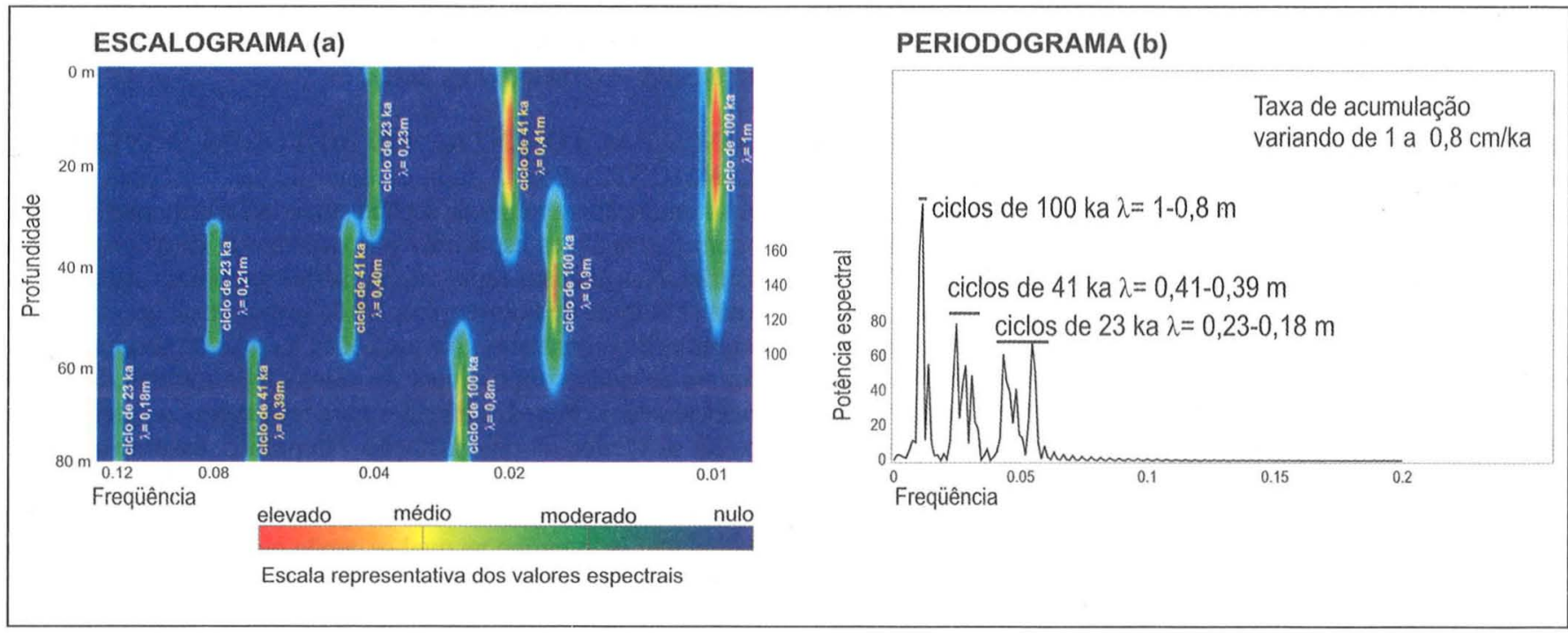

Figura 3 - Análise espectral de uma seção rítmica hipotética com taxa de acumulação variando de 1 a $0,8 \mathrm{~cm} / \mathrm{ka}$ (similar à apresentada na figura 1 b). Como a taxa de acumulação é variável, passando de $1 \mathrm{~cm} / \mathrm{ka}$ para $0,9 \mathrm{~cm} / \mathrm{ka}$, até atingir $0,8 \mathrm{~cm} / \mathrm{ka}$, a espessura dos ciclos varia e a definição das freqüencias no periodograma torna-se baixa. No escalograma as faixas de cor verde, amarelo e vermelho se deslocam ao longo do eixo $(X)$ e $(Y)$. As mudanças na espessura dos ciclos são registradas no eixo $(X)$. Ao longo da escala vertical profundidade (eixo Y) pode-se monitorar a variação da taxa de acumulação ao longo da profundidade, que foi a causa da alteração da espessura dos ciclos.

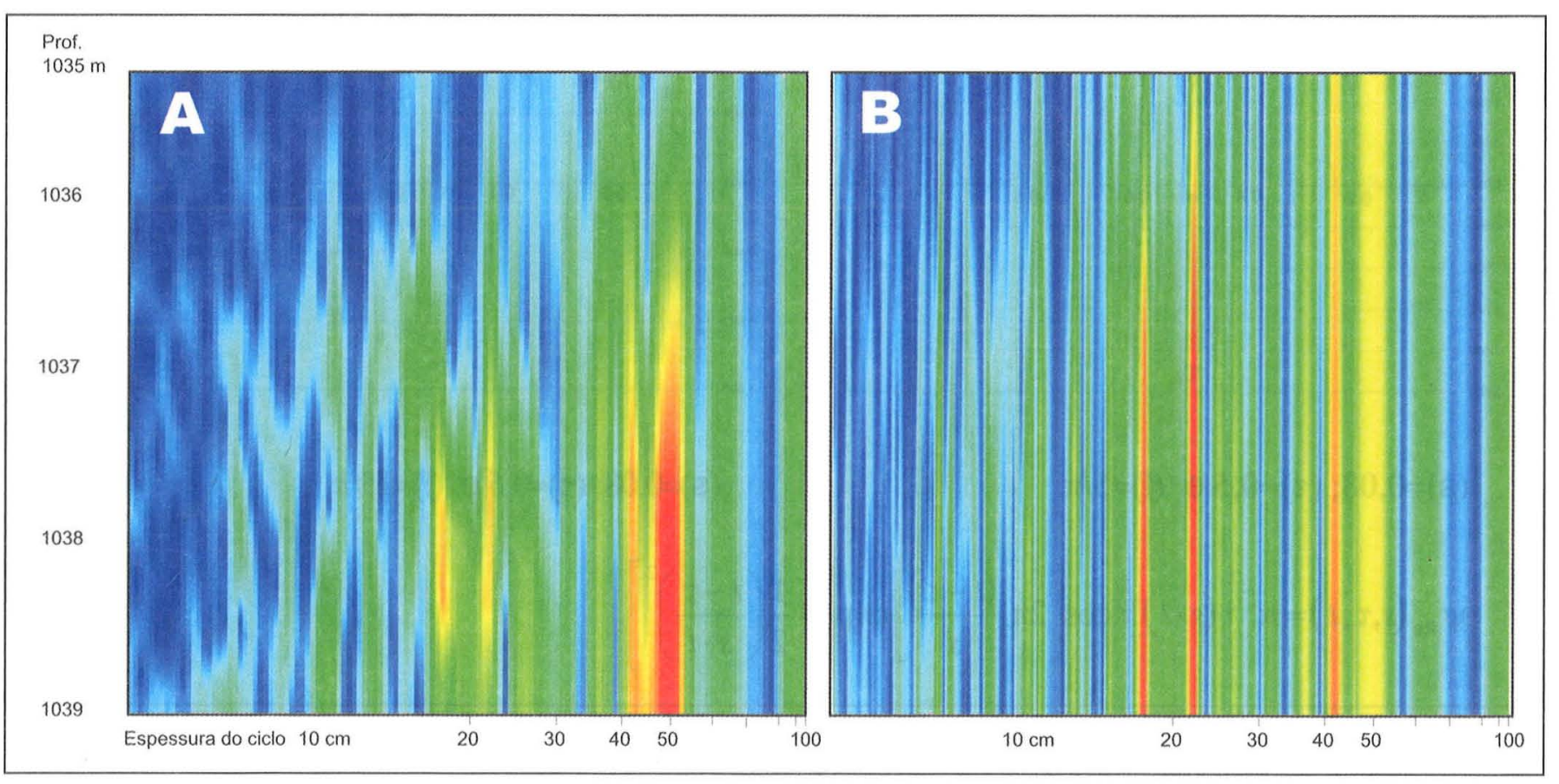

Figura 4 - Os dois escalogramas representam a análise de uma mesma série temporal, a diferença está no parâmetro (l), que no gráfico A foi configurado como $(l)=5$, enquanto em $B(l)=15$. Em ambos foram detectados ciclos de espessura de 19, 23, 43 e $50 \mathrm{~cm}$. Contudo no escalograma A, nota-se que esses ciclos possuem melhor definição segundo a escala vertical de profundidade. Por sua vez, no escalograma B, eles estão representados por uma banda de freqüência mais estreita, sendo mais bem definidos na escala horizontal (periodicidade). 
dade, detectando o agente causador da mudança de periodicidade, ou mesmo do desaparecimento de um determinado ciclo. A figura 3 ilustra a diferença entre escalogramas e periodogramas. Nesta situação hipotética tem-se uma sucessão de estratos escuros e claros cuja taxa de acumulação adquire três valores distintos ao longo da série temporal. Na base da seção a taxa de acumulação foi de $0,8 \mathrm{~cm} / \mathrm{ka}$, passando para $0,9 \mathrm{~cm} / \mathrm{ka}$ e atingindo $1 \mathrm{~cm} / \mathrm{ka}$ no topo. Por meio do escalograma (Fig. 3a) é possível descriminar os três ciclos presentes na série temporal e monitorar as suas respectivas variações no que concerne a freqüência versus profundidade (mudanças de periodicidade ao longo da série temporal). Como discutido anteriormente, no periodograma, principalmente no que se refere aos ciclos menores, além da baixa resolução das freqüências envolvidas, não se tem controle das variações de freqüuência ao longo da série temporal (Fig. 3b).

Além desta vantagem, a Transformada Wavelet permite detectar tanto as freqüências muito baixas como as mais elevadas. Esta característica deve-se a utilização de janelas de análise variável, em contraste com as janelas fixas utilizadas na transformada de Fourier "janelada" (Prokoph \& Barthelmes, 1996). Na prática, o programa ADDSPECTRAL possui somente um parâmetro configurável para tornar possível detectar um espectro de frequiências mais amplo. A configuração adequada do parâmetro (l) permite uma maior resolução no domínio das freqüências ou do tempo, conforme a necessidade da análise. $\mathrm{Na}$ prática, pequenos valores de (l) produzem Wavelets de Morlet de pequeno comprimento de onda, que permitem uma alta resolução na dimensão tempo, porém, baixa resolução em freqüência. A figura 4 exemplifica essa vantagem utilizando um caso real, onde os ciclos de precessão (19 e $21 \mathrm{ka}$ ) e de obliqüidade $(41 \mathrm{ka})$ estão mais bem definidos somente na parte basal da seção estudada. Em ambos escalogramas foram detectadas importantes freqüências que correspondem aos ciclos de comprimento de onda de $19,23,43$ e $50 \mathrm{~cm}$. Contudo no escalograma $A$, onde $1=5$, pode-se notar que esses ciclos são mais bem definidos abaixo da profundidade de $1037 \mathrm{~m}$. Por sua vez, no escalograma $B$, onde $1=15$, eles nos dão a falsa impressão de possuírem um elevado valor espectral ao longo de todo o intervalo analisado. Entretanto como eles são representados por uma banda de freqüência mais estreita é possível discriminar com melhor precisão os ciclos de 40 e $50 \mathrm{~cm}$, e observar outros ciclos com espessuras de 27 a $28 \mathrm{~cm}$.

CICLOESTRATIGRAFIA DO TESTEMUNHO 98 DO POÇO 530 DO DSDP O poço DSDP-530A foi perfurado na Bacia de Angola, cerca de $15 \mathrm{~km}$ ao norte do sopé da Elevação de Walvis e a $150 \mathrm{~km}$ do sopé do talude da margem continental africana, em uma profundidade d'água de $4629 \mathrm{~m}$ (Fig. 5).

A existência de depósitos ricos em matéria orgânica na Bacia de Angola já era conhecida a partir dos resultados obtidos em outros poços do DSDP $(361,363$ e 364$)$. Contudo, a origem destas rochas permanecia relativamente desconhecida sob vários aspectos. Visando preencher essa lacuna no conhecimento da bacia foi perfurado o poço $530 \mathrm{~A}$. Neste poço, no intervalo compreendido entre o Albiano e o Santoniano, foram registradas 260 camadas de folhelhos negros com espessuras variando de 1 a $60 \mathrm{~cm}$ e teores de carbono orgânico (COT) variando entre 1 e 16\% (Hay et al., 1984). Os sucessivos estudos realizados permitem concluir que a seção registra processos periódicos climático-orbitais somados a processos aperiódicos, esses últimos relacionados principalmente a turbiditos (Deroo et al., 1984; Dean et al.,1984; Stow \& Dean, 1984). Além disso, estudos de cicloestratigrafia demonstraram que camadas de fothelhos negros do Cenomaniano-Turoniano seguem um padrão cíclico da ordem de 19 ka a 100 ka (Fig. 6). A origem destes depósitos deve-se à alternância de períodos úmidos e secos modulados por mecanismos climático-orbitais. Estas mudanças climáticas ocorreriam tanto na escala sazonal, formando lâminas milimétricas, como em uma escala compatível com os ciclos de Milankovitch (Cunha, 2001).

Para testar as diferenças nos resultados obtidos por meio da análise espectral utilizando o programa Statistica ${ }^{\mathrm{TM}}$ (FFT) e o programa ADDSPECTRAL (Wavelet) realizourse a análise espectral do índice colorimétrico que representa sucessão rítmica de argilitos e folhelhos negros do testemunho 98 (1035-1040 m) do poço DSDP 530A. Denomina-se neste estudo de índice colorimétrico os dados numéricos que representam as variações de cores das litologias recuperadas no testemunho. Atualmente, existe um grande número de estudos cicloestratigráficos realizados em séries temporais que representam as variações de cores, comprovando a eficiência destas técnicas (Busch, 1991; Molinie \& Ogg, 1992; Sageman et al.,1997; Yang \& Kominz, 1999; Balsam et al., 1999). A análise espectral das curvas que expressam o índice colorimétrico segue o método descrito em Sageman et al. (1997), com algumas adaptações. Segundo este método as oscilações de cores observada nos testemunhos, resultante da alternância de lâminas escuras e claras, podem ser transformadas em uma seqüência numérica. A imagem em tons de cinza do testemunho é escaneada e convertida por meio de um programa de computador em uma matriz numérica. Esta matriz é composta por números que oscilam de zero, algarismo que representa a cor preta, a 255, valor correspondente à cor branca. Os números intermediários entre o número 1 e o 254 codificam 254 tons de cinza. Esta matriz é simplificada matematicamente em uma matriz com uma única coluna, tornando-se uma série temporal representativa das variações de cor do testemunho. Balsam et al., (1999) comprovam que este método pode ser utilizado para avaliar as variações composicionais da rocha, desde que balizado por análises geoquímicas e físicas (grau de umidade, teor e tipo de argila, quantidade de $\mathrm{MO}$ e óxidos de metais). $\mathrm{O}$ controle composicional das bandas claras e escuras foi feito por meio da medição de carbono orgânico total (COT).

Análise espectral comparativa: Wavelet versus Harmônicos O periodograma referente aos testemunhos 98 possui alto valor espectral para duas faixas de frequiências que são $38-61 \mathrm{~cm}$ e $19-23 \mathrm{~cm}$ (Fig. 7b). Existem ainda alguns valores com elevado potencial espectral isolados destas duas faixas, que correspondem a ciclos de espessura de $8,12,26$ e $33 \mathrm{~cm}$.

Os ciclos em destaque nestes testemunhos podem ser associados aos ciclos orbitais de precessão e de obliqüidade. No testemunho 98 , o principal ciclo em destaque é o de $1=49 \mathrm{~cm}$, que associado à obliqüidade resultaria em uma taxa de acumulação de $1,2 \mathrm{~cm} / \mathrm{ka}$. Já os ciclos de $21 \mathrm{ka}$ e $19 \mathrm{ka}$, relacionam-se aos ciclos $1=23 \mathrm{~cm} \mathrm{e} 1=19 \mathrm{~cm}$, fornecendo taxas de acumulação de 0,99 e $1 \mathrm{~cm} / \mathrm{ka}$, respectivamente. Os ciclos de $1=8-12 \mathrm{~cm}$, considerando um valor médio de $10 \mathrm{~cm}$ poderiam estar relacionados ao ciclo orbital reconhecido por Park et al. (1993) como um ciclo de cerca de $11 \mathrm{ka}$, denominado de "double beat" (Fig. 7b). Em teoria este ciclo é registrado freqüentemente em regiões próximas ao equador. Contudo devido a correntes oceânicas esta indução pode causar efeitos em áreas mais distantes.

$\mathrm{Na}$ análise espectral do testemunho 98 utilizando o método de Transformada da Wavelet de Morlet, observa-se um alto valor espectral para o ciclo de comprimento de onda de 49 $\mathrm{cm}$, seguida de uma faixa de valores espectrais elevados para as freqüências relativas aos ciclos de $1=41,23$ e $19 \mathrm{~cm}$. Em um grau de intensidade menor observam-se ainda freqüencias relacionadas aos ciclos de $1=11$ e $12 \mathrm{~cm}$. Esses resultados são similares aos obtidos no periodograma. Os ciclos de $1=41 ; 23$ e $19 \mathrm{~cm}$ são correlacionáveis com os ciclos de obliqüidade (41 ka) e precessão (23 e $19 \mathrm{ka}$ ), com taxas de acumulação em torno 


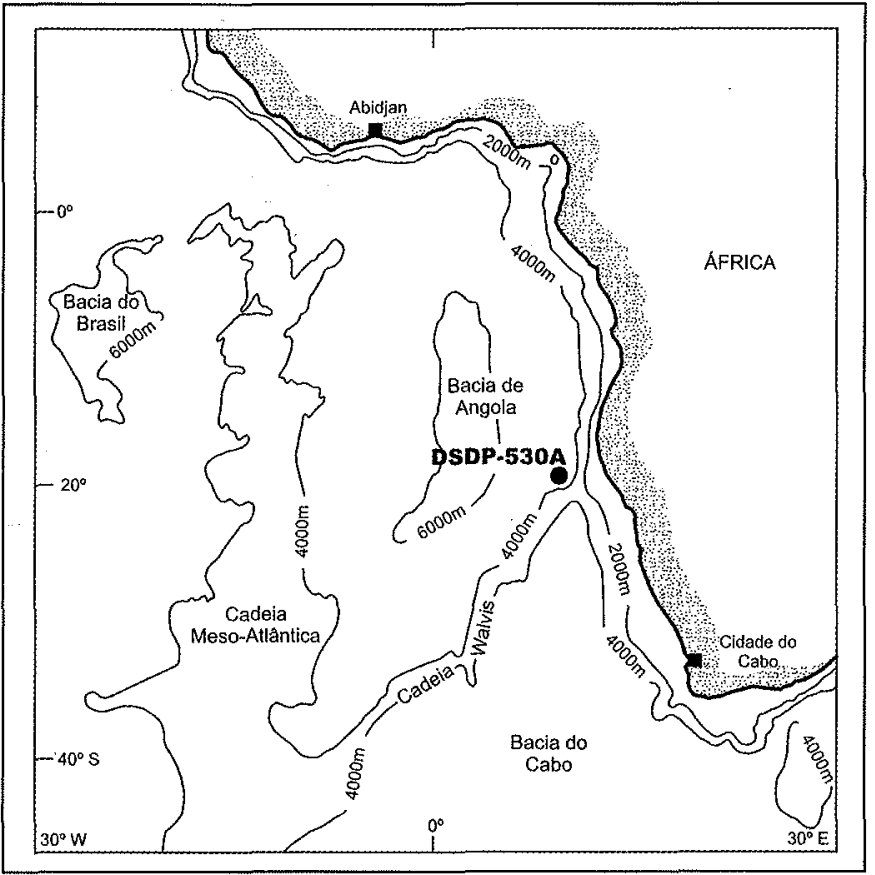

Figura 5 - Mapa de localização do poço DSDP-530A.

de $1 \mathrm{~cm} / \mathrm{ka}$.

Apesar dos resultados obtidos a partir dos dois métodos não diferirem substancialmente, algumas inferências somente são possíveis de serem feitas analisando o escalograma: A) A informação mais importante, obtida exclusivamente no escalograma, é que os ciclos que estão bem definidos no periodograma, são exclusivos do intervalo entre 1037,5 e $1039,5 \mathrm{~m}$. No intervalo inicial e final do testemunho a obliteração dos sinais cíclicos climáticos pode estar relacionada a turbiditos não periódicos, ou intensa bioturbação, destruindo o padrão rítmico das rochas. Visualmente, na figura 6 observa-se que as camadas de folhelhos negros são mais freqüentes no intervalo superior, porém de distribuição mais caótica. B) O ciclo de $1=49 \mathrm{~cm}$, utilizando apenas o periodograma, poderia ser interpretado como associado ao ciclo de obliqüidade, alterações na taxa de acumulação ao longo da seção poderiam ter causado a mudança na espessura de ciclo que oscilariam de $\mathrm{l}=38 \mathrm{a} 61 \mathrm{~cm}$ de espessura. Ciclos maiores e menores que os de $1=23-19 \mathrm{~cm}$ poderiam respaldar essa interpretação. Contudo, a análise do escalograma demonstra que o ciclo de espessura igual a $49 \mathrm{~cm}$ e $41 \mathrm{~cm}$ são concorrentes em tempo, conseqüentemente à explicação de uma causa comum associada apenas à variação da taxa de acumulação não é plausível. A presença destes dois ciclos deve-se, provavelmente, à existência de padrões' de ciclicidade distintos, induzidos por causas independentes. C) Os ciclos de espessura menor que 1 $=19 \mathrm{~cm}$, alguns deles reconhecidos no periodograma e inicialmente interpretados como relacionáveis aos ciclos orbitais de $11 \mathrm{ka}$, aparecem no escalograma como ciclos de valor espectral reduzido e pouca continuidade ao longo do intervalo analisado (Fig. 7a). Esse comportamento permite interpretar que eles são produto da interferência de ciclos periódicos de curta duração temporal, possivelmente associados aos turbiditos. Turbiditos síltico-argilosos, ocorrendo com frequêencia desde 8 a $20 \mathrm{ka}$ foram descritos neste poço e em outros da costa da África ao Sul (Dean et al., 1978 e Stow, 1984).

CONCLUSÕES Nos estudos cicloestratigráficos o uso da técnica de análise espectral fundamentada na Transformada Wavelet de Morlet pode ser mais vantajoso se comparada às téc-

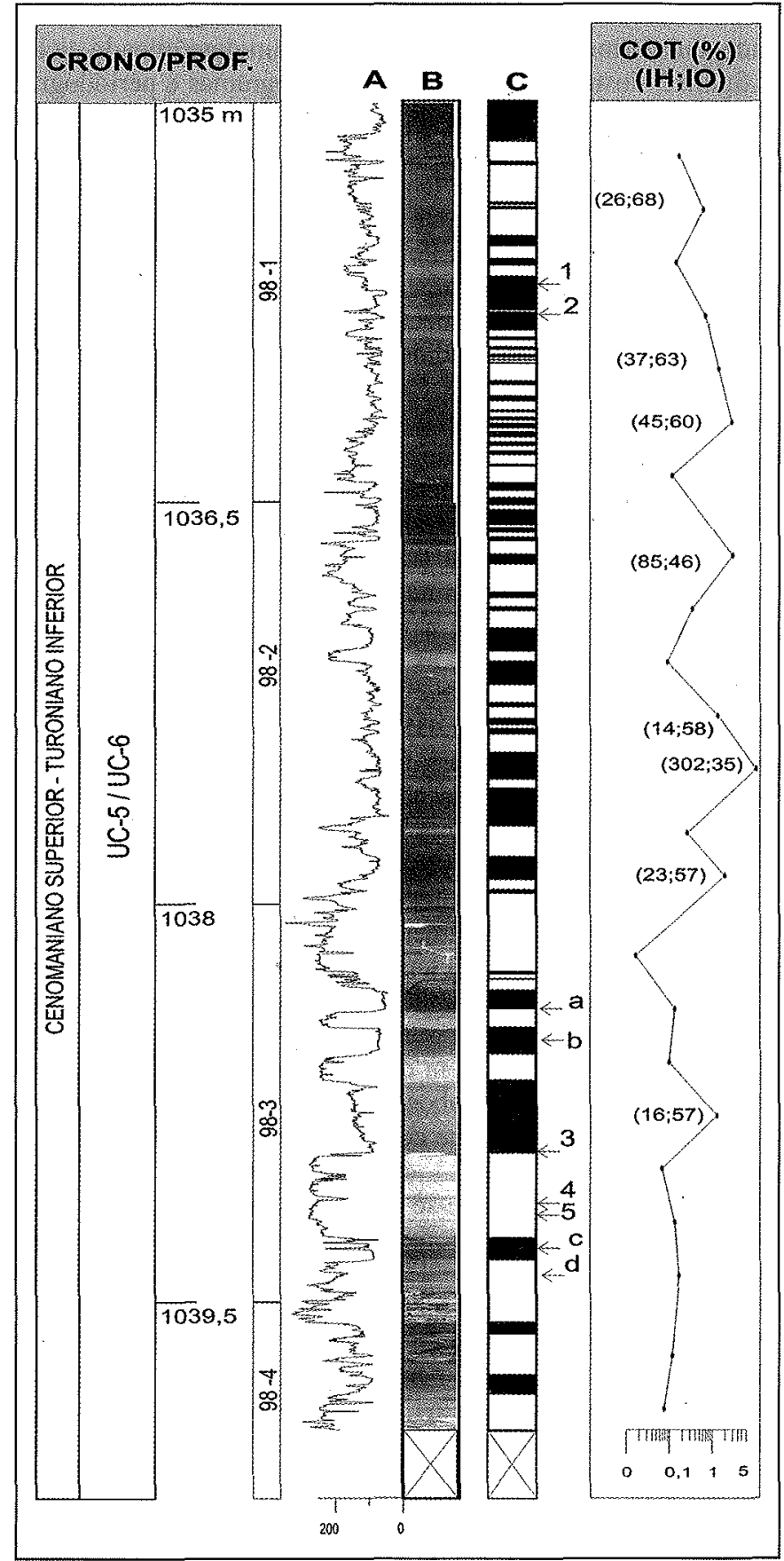

Figura 6 - Perfil do índice colorimétrico (A), foto (B), posição dos folhelhos negros $(C)$ e resultados das análises geoquimicas e biocronoestratigráficas (Cunha, 2001) do testemunho 98 do poço DSDP-530A. O testemunho é caracterizados por camadas de argilitos - lamitos esverdeados (5G 6/1 e $5 G$ 4/1), bioturbados e folhelhos negros (N2-N3) piritizados e laminados. Ambas litologias eventualmente apresentam estruturas sedimentares de turbiditos síltico-argilosos.

nicas que utilizam a Transformada de Fourier. O monitoramento das variações do conteúdo de freqüência ao longo da dimensão tempo ou profundidade permite obter interpretações mais precisas sobre a origem dos ciclos presentes nas séries temporais. Outra vantagem desta técnica sobre a utilização de harmônicos é o dimensionamento da janela de análise. $O$ correto dimensionamento pode aumentar a resolução no campo das frequêencias em detrimento da localização do sinal, ou inversamente, permite uma localização precisa do sinal na série temporal com conse- 


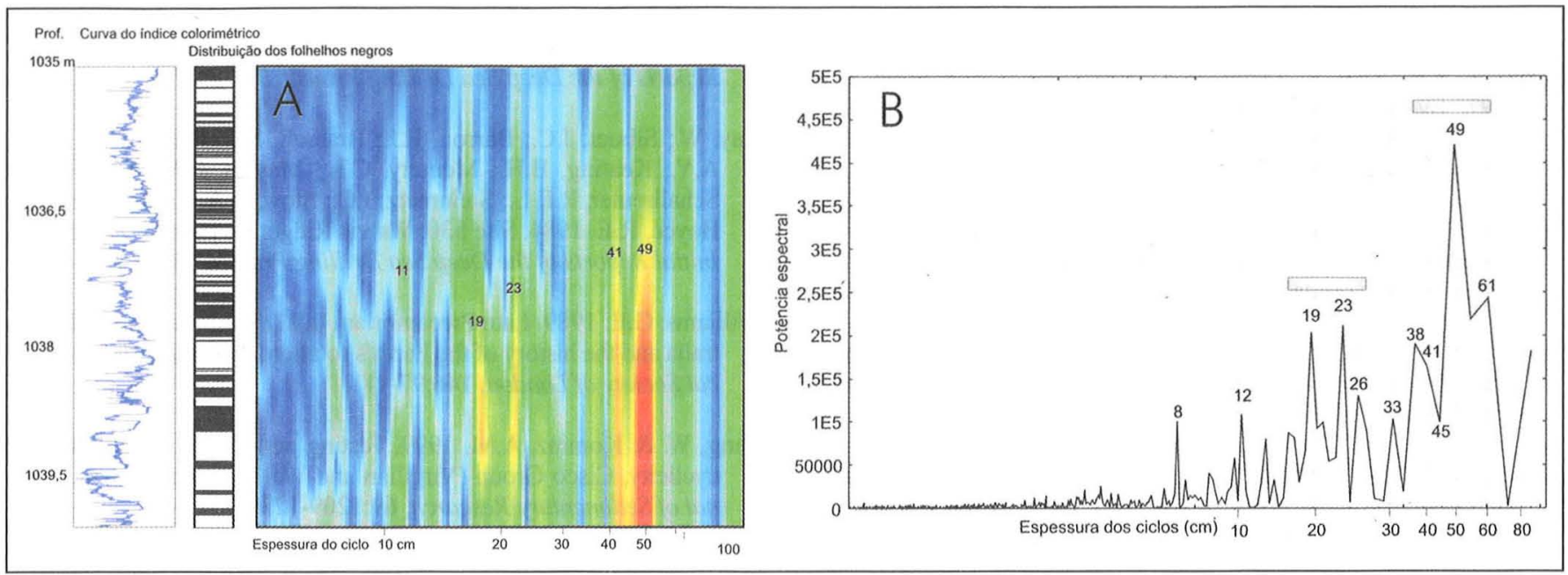

Figura 7 - Comparação dos resultados obtidos nas análises espectrais por meio da transformada de Fourier e Wavelet. No escalograma pode-se observar que os principais ciclos detectados na análise feita por harmônicos (periodogramas), que possuem espessura nas faixas de $l=38-61 \mathrm{~cm}$ e $19-23 \mathrm{~cm}$, são restritos ao intervalo 1037,5 e 1039,5 $\mathrm{m}$.

qüente perda da qualidade da resolução das freqüências. Essa vantagem permite pesquisar na série temporal freqüências de diferentes dimensões com maior eficiência. A desvantagem principal da técnica de Wavelet é a falta de programas industriais de aplicação simples, como por exemplo o Statistica ${ }^{\mathrm{TM}}$, amplamente utilizado para a análise de harmônicos devido a suas inúmeras facilidades. A parametrização da janela de análise de forma a obter os melhores resultados também dificulta a utilização das técnicas que usam Wavelet, tornando os métodos de Fourier mais expeditos. O caso de estudo apresentado neste trabalho, a análise do testemunho 98 do poço 530A do DSDP, demonstrou que a análise que utiliza Wavelet é eficiente deste que a parametrização da janela de análise seja feita corretamente. Os resultados obtidos caso seja feita uma parametrização inadequada serão muito similares aos obtidos na Análise Rápida de Fourier, ou seja o monitoramento dos ciclos ao longo do intervalo de profundidade estudado poderá não ser obtido. Assim mesmo a definição do conteúdo de freqüência parece ser mais precisa quando obtida através dos métodos que utilizam Wavelet.

Agradecimentos Os autores agradecem a PETROBRAS pela permissão para publicação deste trabalho. Ao ADDLAB da Universidade Federal Fluminense que desenvolveu em conjunto com o CENPES/PETROBRAS o programa ADDSPECTRAL. Gostaríamos ainda de agradecer a geóloga Trícia da Silva Mello que durante o estágio realizado no CENPES-BPA ficou responsável pelos testes para verificar a eficiência do programa ADDSPECTRAL durante a fase de desenvolvimento.

\section{Referências}

Balsam, W.L.; Deaton, B.C. \& Damuth, J.E. 1999. Evaluating optical lightness as a proxy for carbonate content in marine sediment cores. Marine Geology, 161:141-153.

Busch, W.H. 1991. Analysis of wet-bulk density and sediment color cycles in Pliocene-Pleistocene sediments of the Owen Ridge (Site 722) and Oman Margin (Site 728). In: W.J Prell \& N. Niitsuma (eds.). Proceedings of the Ocean Drilling Program, Scientific Results, 117: 239-253.

Cunha, A. A. S. 2001. Cicloestratigrafia no Cenomaniano superior e Turoniano do Oceano Atlântico Sul. Tese de Doutorado, Instituto de Geociências, Universidade Federal do Rio Grande do Sul, 509 p.

Dean, W.E.; Arthur, M.A. \& Stow, D.A.V. 1984. Origin and geochemistry of Cretaceous deep, sea black shales and multicolored claystones, with emphasis on Deep Sea Drilling Project Site 530, southern Angola Basin. In: Hay, W. et al.(eds.). Initial Reports of the Deep Sea Drilling Project, 75:819-844.

Dean, W.E.; Gardner, J.V.; Jansa, L.F.; Cepek, P. \& Seibold, E. 1978. Cyclic sedimentation along the continental margin of Northwest Africa. In: J.V Gardner \& J. Herring, (eds.). Initial Reports of the Deep Sea Drilling Project, 41: 965-989.

Deroo, G.; Herbin, J.P. \& Huc, A.Y. 1984. Organic geochemistry of
Cretaceous black shales from Deep Sea Drilling Project Site 530, Leg 75, eastern South Atlantic. In: Hay, W. et al.(eds.). Initial Reports of the Deep Sea Drilling Project, 75:983-999.

Molinie, A.J. \& Ogg, J.G. 1992. Milankovitch cycles in Upper Jurassic and Lower Cretaceous radiolarites of the Equatorial Pacific: spectral analysis and sedimentation rate curves. In: R.L. Larson; Y. Lancelot et al. (eds.). Proceedings of the Ocean Drilling Program, 129:529-547. (Scientific results).

Niebuhr, B. \& Prokoph, A. 1997. Periodic-cyclic and chaotic successions of Upper Cretaceous (Cenomanian to Campanian) pelagic sediments in the North German Basin. Cretaceous Research, 18:731-750.

Park, J.; D’hondt, S.L.; King, J.W. \& Gibson, C. 1993. Late Cretaceous Precessional Cycles in Doble Time: A Warm-Earth Milankovitch Response. Science, 261:1431-1434.

Prokoph, A. \& Agterberg, F.P. 1999. Detection of sedimentary cyclicity and stratigraphic completeness by wavelet analysis: An application to late Albian cyclostratigraphy of the western Canada sedimentary basin. Journal of Sedimentary Research, 69: 862-875.

Prokoph, A. \& Barthelmes, F. 1996. Detection of nonstationarities in geological time series: wavelet transform of chaotic and cyclic se- 
quences. Computers \& Geosciences, 22:1097-1108.

Sageman, B.B.; Rich, J.; Arthur, M.A.; Birchfield, G.E. \& Dean, W.E. 1997. Evidence for Milankovitch Periodicities in CenomanianTuronian lithologic and Geochemical Cycles, Wester Interior U.S.A. Journal of Sedimentary Research, 67: 286-302.

Sprenger, A. \& Ten Kate, W.G. 1993. Cross-spectral analysis of two late Berriasian rhythmic limetones-marl sucessions in SE Spain and SE France favours orbital control. Geologie en Mijnbouw, 72:69-83.

Stow, D.A.V. \& Dean, W.E. 1984. Middle Cretaceous black shales at Site 530 in the southeastern Angola Basin. In: Hay, W. et al.(eds.). Initial Reports of the Deep Sea Drilling Project, 75:809-817.

Stow, D.A.V. 1984. Turbidite facies, associations, and sequences in the southeastern Angola Basin. In: W. Hay; J.C. Sibuet; E.J. Barron; S.C. Brassell; W.E. Dean; A.Y. Huc; B.H. Keating; C. L. Mcnul- ty; P.A. Meyers; M. Nohara; R.E.L. Schallreuter; J.C Steinmetz; D.A.V. Stow; H. Stradner; R.E. Boyce \& R. Amidei (eds.). Initial Reports of the Deep Sea Drilling Project, 75:785-799.

Hay, W.; Sibuet, J.C.; Barron, E.J.; Brassell, S.C.; Dean, W.E.; Huc, A.Y.; Keating, B.H.; Mcnulty, C.L.; Meyers, P.A.; Nohara, M.; Schallreuter, R.E.L.; Steinmetz, J.C.; Stow, D.A.V.; Stradner, H. \& Boyce, R.E. 1984. Site 530; Walvis Ridge. In: Hay, W. et al.(eds.). Initial Reports of the Deep Sea Drilling Project, 75:29-285.

Williams, G.E. 1989. Late Precambriam tidal rhythmites in South Australia and the history of the Earth's rotation. Journal of the Geological Society of London, 146:97-111.

Yang, W. \& Kominz, A.M. 1999. Testing periodicity of deposicional cyclicity, Cisco Group (Virgilian and Wolfcampian), Texas. Journal of Sedimentary Research, 69:1209-1231. 